Presented at the 4 th Conference on Intersections between Particle and Nuclear Physics, Tucson, Arizona, May 1991.

\title{
DUMAND-II Progress Report
}

\section{Presented by R. J. Wilkes, for The DUMAND Collaboration:}

R. Becker-Szendy ${ }^{4}$, P. Bosetti ${ }^{1}$, J. Bolesta ${ }^{4}$, P.E. Boynton ${ }^{14}$, H. Bradner ${ }^{9}, U$. Camerini ${ }^{15}$, S.T. Dye ${ }^{3}$, P.K.F. Grieder ${ }^{2}$, T. Hayashino ${ }^{10}$, E. Hazen ${ }^{3}$, M. Ito ${ }^{10}$, H. Kawamoto ${ }^{10}$, T. Kitamura ${ }^{7}$, K. Kobayakawa ${ }^{6}$, P. Koske ${ }^{5}$, J.G. Learned ${ }^{4}$, C. Ley ${ }^{1}$, J.J. Lord ${ }^{14}$, R. March ${ }^{15}$, S. Matsuno ${ }^{4}$ K. Miller ${ }^{13}$, P. Minkowski ${ }^{2}$, K. Mitsui ${ }^{11}$, D. O'Connor ${ }^{4}$, Y. Ohashi ${ }^{11}$, A. Okada ${ }^{11}$, V.Z. Peterson ${ }^{4}$, A. Roberts ${ }^{4}$, C.E. Roos ${ }^{13}$, M. Sakuda ${ }^{12}$, D. Samm ${ }^{1}$, V. Stenger ${ }^{4}$, H. Suzuki ${ }^{10}$, S. Tanaka ${ }^{10}$, S.

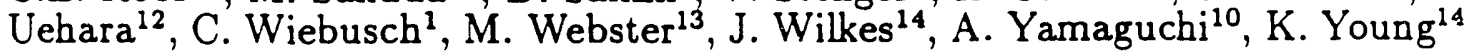

1) Techn. Hochschule Aachen, Germany; 2) Univ. of Bern, Switzerland; 3) Boston Univ., USA; 4) Univ. of Hawaii, USA; 5) Univ. of Kiel, Germany; 6) Kobe Univ., Japan; 7) Kinki Univ., Japan; 8) Okayama Science Univ., Japan; 9) Scripps Inst. of Oceanography, USA; 10) Tohoku Univ., Japan; 11) ICRR, Univ. of Tokyo, Japan; 12) KEK, Tsukuba, Japan; 13) Vanderbilt Univ., USA; 14) Univ. of Washington, USA; 15) Univ. of Wisconsin, USA.

Abstract: The design, scientific goals, and capabilities of the DUMAND II detector system are described. Construction was authorized by DOE in 1990, and development of various detector subsystems is under way. Current plans include deployment of the shore cable, junction box and three strings of optical detector modules in 1992, with expansion to the full 9-string configuration about one year later.

DUMAND-II is a water Cerenkov detector for high energy astrophysical neutrinos. Nine vertical strings each contain 24 hemispherical photomultiplier tubes, for a stal complement of 216 Optical Modules (PMTs and associated fast electronics in glass pressure housings). The ocean bottom array is sited approximately $30 \mathrm{~km}$ west of Keahole Point on the Island of Hawaii, at a depth of 4.7 $\mathrm{km}$. A cable to shore, terminating in the Junction Box to which the strings are connected, provides power and fiber optic data communications. The DUMANDI experiment $[1,2]$ operated a short prototype string near this site, and confirmed the ability of the planned system to detect and reconstruct muon tracks.

The strings are located at the corners of an equilateral octagon, with the ninth string at its center. The active volume of the array will have a height of $230 \mathrm{~m}$ and a diameter of $105 \mathrm{~m}$, with the lowest module $100 \mathrm{~m}$ above the ocean floor. Horizontal and vertical spacings between the modules are $40 \mathrm{~m}$ and $10 \mathrm{~m}$, respectively. Array geometry has been optimized for the detection of high energy ( $>25 \mathrm{GeV}$ ) muons from neutrino interactions, as well as contained neutrino induced cascades. Muons will be detected with high efficiency and reconstructed in direction with a median accuracy of about $1^{\circ}$. The scattering angle between the incident neutrino and the resulting muon will be within this error for neutrino energies above about $1 \mathrm{TeV}$. The site places no significant constraints on future expansion of the system.

There will be a high rate in the downward direction (zenith angles $\theta<80^{\circ}$ ) due to cosmic ray muons (decay products of cosmic ray secondaries which are sufficiently energetic to penetrate the water overburden). For zenith angles $>$ $80^{\circ}$, the observed flux will be dominated by neutrinos produced in cosmic ray interactions in the atmosphere. For zenith angles beyond $80^{\circ}$, with solid angle $2.35 \pi$ steradians, the rate for atmospheric neutrino induced muon events will be about 4500 per year, i.e., a background of about one event per $\left(2.3^{\circ}\right)^{2}$ ptr year. Thus, with the design resolution of 1 degree for muon direction reconstruction, 
we will be largely signal limited, rather than background limited, in the search for extraterrestrial point sources.

We expect detectable signals in DUMAND II from a number of point sources both inside and outside the galaxy. Recently, a new model for ultrahigh energy neutrino production in Active Galactic Nuclei (AGN) has been described[3]. Taking into account the summed flux from all $A G N$, calculations suggest that for muon energies above about $50 \mathrm{GeV}$, AGNs may be the dominant source of neutrinos. Muon detection rates in DUMAND for atmospheric and AGN neutrinos are shown in figure la as a function of detected muon energy; in figure $1 \mathrm{~b}$ we show the expected zenith angle distribution. Note that atmospheric and AGN neutrinos have similar angular distributions for very different reasons: the atmospheric $\nu$ rate peaks near the horizon due to the pion decay path distribution, while the AGN rate is depressed at the nadir because the very high mean energy causes significant attenuation in the earth. If the fluxes predicted in ref. [3] are observed, we would expect about 3500 neutrinos events per year from AGNs, nearly half with energy over $10 \mathrm{TeV}$ at the detector[4]. At these rates, the angular distribution might permit a $5 \%$ determination of the earth's core d.nsity in one year.

DUMAND will complement accelerator research, since no currently existing or planned accelerator facility produces neutrino beams above about $600 \mathrm{GeV}$. The AGN model predicts substantial $\nu_{e}$ fluxes which may permit the observation of resonant $W^{-}$production at $6.4 \mathrm{PeV}[4]$. A proposal to Fermilab (P824) requests that construction of the new $150 \mathrm{GeV}$ proton injector include facilities for directing a neutrino beam toward the DUMAND site to permit long baseline neutrino oscillations studies.

DUMAND will provide unprecedented opportunities for long term deep ocean observations. Hydrophones on the strings will digitize acoustical signals up to $100 \mathrm{kHz}$. Since the cable provides a high-rate data link to the shore station, sophisticated realtime processing of acoustical signals can be employed, to ensure accurate OM location and to observe the acoustical background. These data can also be used to explore the possibility of acoustic detection for ultrahigh energy neutrinos.

Construction of the shore cable is under way, and deployment of the cable and its terminating Junction Box (along with associated environmental monitoring equipment) is planned for mid 1992, followed later in the year by deployment and connection of the first three strings. The shore station facilities at Keahole Point are in the design stage, with construction to begin in early 1992 . OMs using both Hamamatsu and Phillips photomultipliers have been prototyped and tested, including a recent underwater test of Phillips units near the Canary Islands. Critical fast logic designs for the String Controllers have been finalized. Workshops on triggering and ocean engineering aspects of DUMAND held recently in Madison and Seattle indicate that development of the various subsytems is proceeding on schedule.

Acknowledgements: We are indebted to the US Department of Energy, the US National Science Foundation, the Japanese Ministry of Science, the Japan Society for the Promotion of Science, and the Japanese and Swiss National Science Foundations for supporting this project.

Rếéreñces:

1. S. Matsuno, et al, N.I.M., A276 (1989) 359.

2. J. Babson, et al, Physical Review D42 (1990) 3613.

3. F. W. Stecker, C. Done, M. H. Salamon and P. Sommers, "Ultrahigh Energy 
Neutrinos from Active Galactic Nuclei and their Detectability", Univ. of Utah preprint (to be published in Proc. of 3rd Int. Workshop on Neutrino Telescopes, Venice, 1991).

4. J. G. Learned and T. Stanev, "Detection of Neutrinos from Active Galactic Nuclei", Univ. Hawaii Preprint HDC-1-91, (to be published in Proc. of 3rd Int. Workshop on Neutrino Telescopes, Venice, 1991).

Figure 1: Calculated muon rates in the DUMAND-II array from atmospheric and AGN neutrino events, as a function of the a) muon energy; b) muon zenith angle $\theta_{\mu}$.

a)

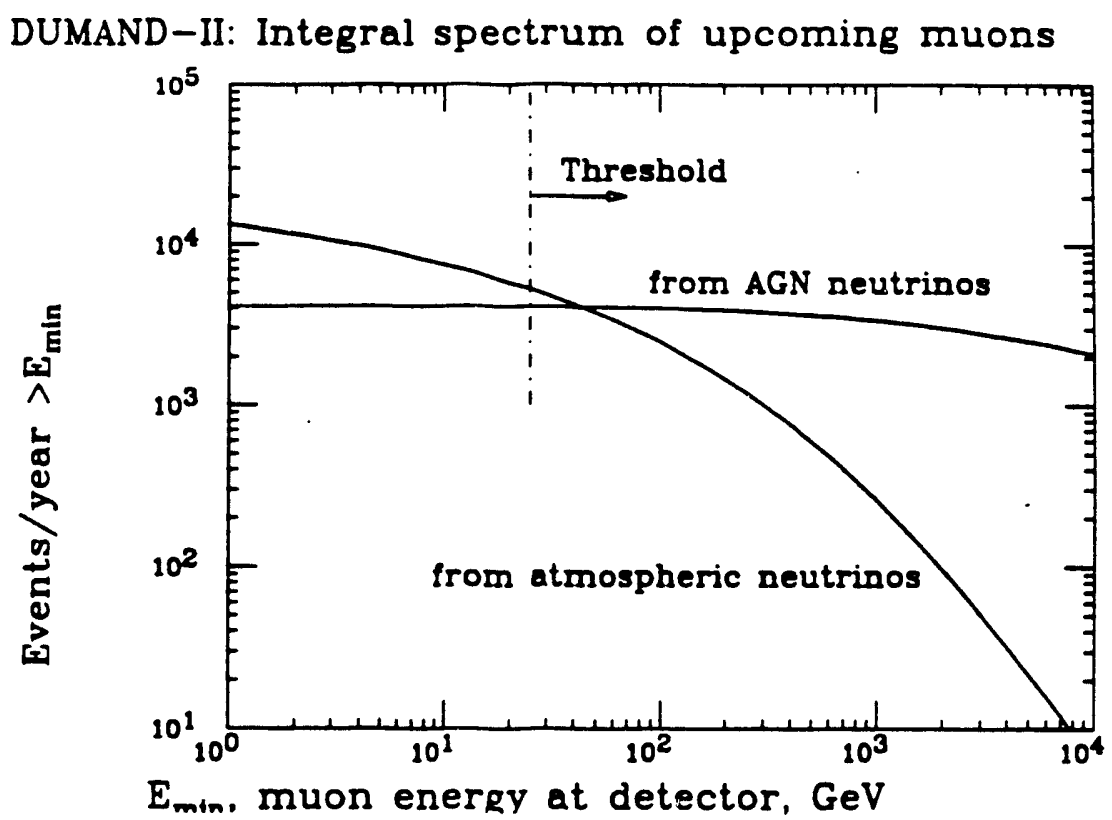

b)

DUMAND-II: Muon angular distributions

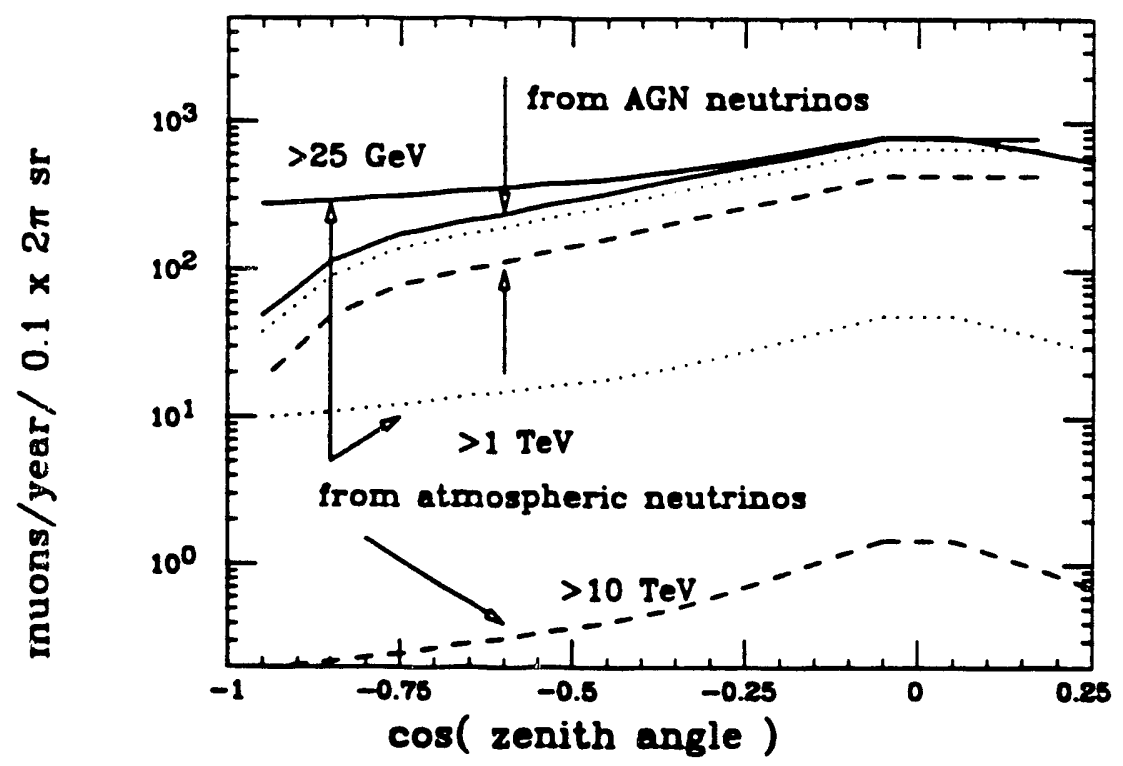




\section{DISCLAIMER}

This report was prepared as an account of work sponsored by an agency of the United States Government. Neither the United States Government nor any agency thereof, nor any of their employees, makes any warranty, express or implied, or assumes any legal liability or responsibility for the accuracy, completeness, or usefulness of any information, apparatus, product, or process disclosed, or represents that its use would not infringe privately owned rights. Reference herein to any specific commercial product, process, or service by trade name, trademark, manufacturer, or otherwise does not necessarily constitute or imply its endorsement, recommendation, or favoring by the United States Government or any agency thereof. The views and opinions of authors expressed herein do not necessarily state or reflect those of the United States Government or any agency thereof. 

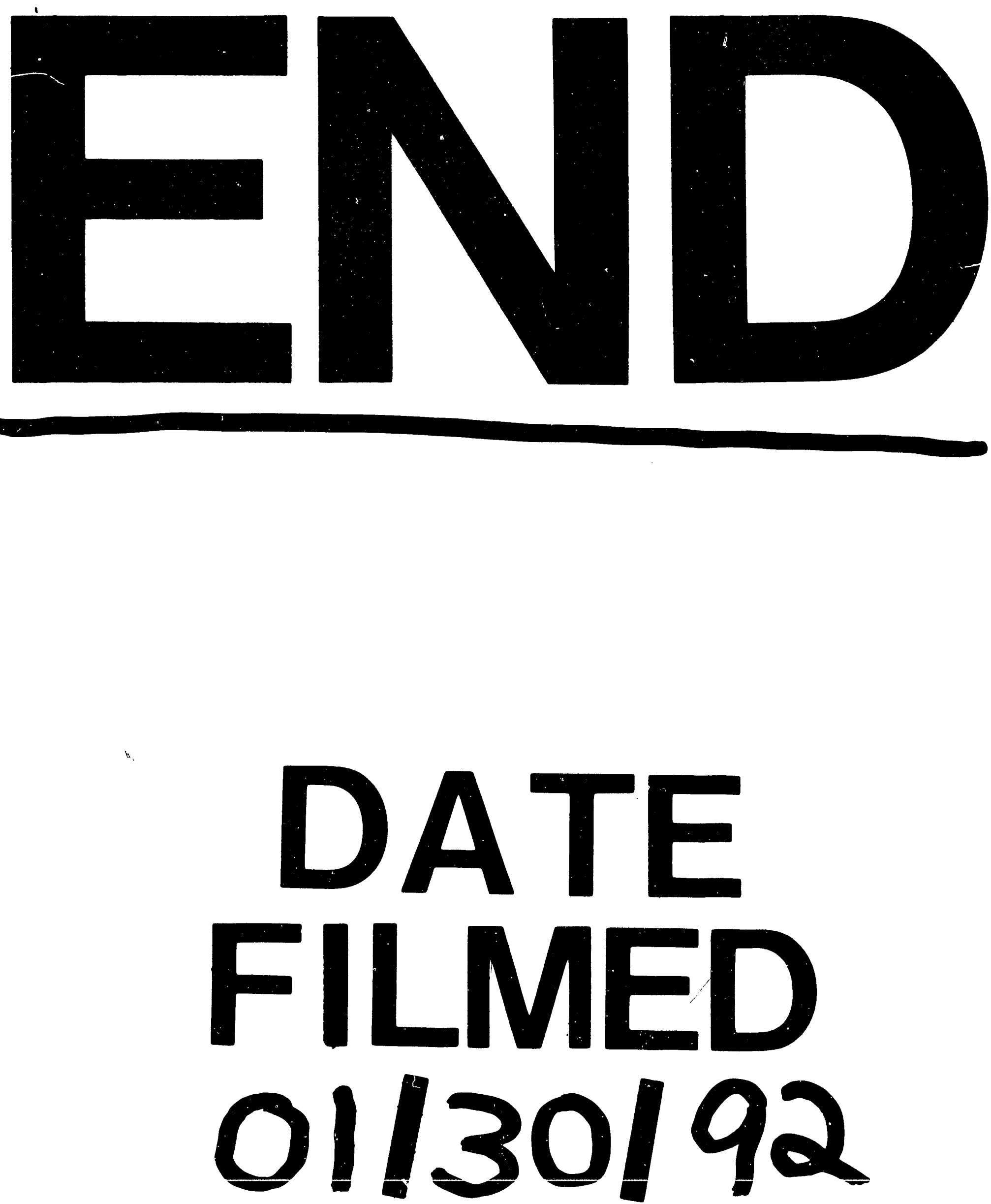

$I$ 


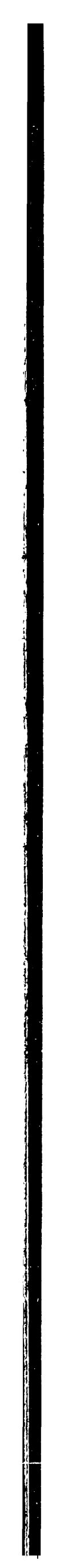

